\title{
The Stille Reaction: Applications in the Synthesis of Organic Dyes for DSSCs
}

\author{
Gianna Reginato*a ${ }^{\star}$ Alessandro Mordini ${ }^{\star a b}$ Lorenzo Zani ${ }^{a}$, Massimo Calamante ${ }^{\mathrm{ab}}$, Alessio Dessì ${ }^{\mathrm{a}}$, \\ and Daniele Franchiab
}

\begin{abstract}
The cross-coupling reaction of organic electrophiles with organostannanes, traditionally known as the Stille reaction, has found renewed interest in the preparation of new organic materials such as conjugated polymers, organic semiconductors and photoactive molecules for use in organic photovoltaics. Moreover, a very recent field in which the Stille reaction has found successful application is that of the design and synthesis of new photosensitizers for dye-sensitized solar cells (DSSCs). DSSCs are considered a promising alternative for energy production from renewable sources. In such devices light harvesting is carried out by a dye which is generally a highly conjugated molecule. Due to the mild operating conditions and the high functional-group compatibility, the Stille reaction proved to be a powerful tool not only for the preparation of photosensitizers, but also to plan their chemical elaboration in order to tune and optimize their photophysical, electrochemical and photovoltaic properties. In this microreview some recent examples of the Stille reaction in the synthesis of organic dyes for DSSC are reported.
\end{abstract}

Keywords: DSSC · Organic dyes · Organostannanes · Stille reaction

\section{Introduction}

The cross-coupling reaction of organic electrophiles with organostannanes, traditionally known as the Stille reaction (Fig. 1), was discovered in the late seventies $^{[1]}$ and rapidly developed as a highly versatile methodology which has been widely used for the synthesis of functional molecules and polymers, both in research laboratories and industry. ${ }^{[2]}$

Despite the often claimed drawbacks connected with tin toxicity, contamination and low atom economy, the Stille coupling still has its own position in cross-couplings as demonstrated by a wide number of very elegant and efficient examples, especially in the generation of carbocyclic and heterocyclic rings, five- and six-membered or macrocyclic systems, or in the total synthesis of natural products. ${ }^{[3]}$ The reaction is a mild process with some remarkable qualities: first of all, organostannanes are accessible by numerous methods and are

${ }^{*}$ Correspondence: Dr. G. Reginato; Dr. A. Mordini

E-mail: gianna.reginato@iccom.cnr.it;

alessandro.mordini@unifi.it

alstituto di Chimica dei Composti Organometallici (CNR-ICCOM)

Via Madonna del Piano 10

50019 Sesto Fiorentino, Italy

bDepartment of Chemistry "U. Schiff"

Università degli Studi di Firenze

Via della Lastruccia 13, 50019 Sesto Fiorentino, Italy

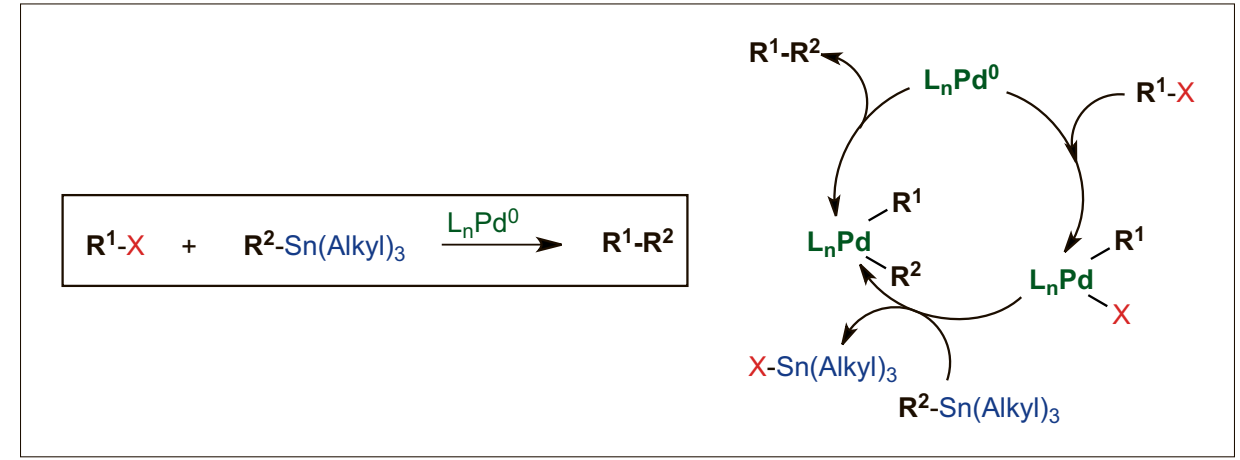

Fig. 1. The Stille reaction and its simplified catalytic cycle. relatively insensitive to moisture and oxygen. Furthermore, due to their low basicity and high steric demand, they are less nucleophilic and less reactive than other organometallic coupling counterparts, thus highly desirable when selective processes and functional-group compatibility are required. Many improvements of the procedure have been attained in recent years, particularly concerning the mechanistic understanding, ${ }^{[3]}$ the development of new and more efficient catalytic systems with lower loadings, ${ }^{[4]}$ and the use of mild con- ditions such as environmentally friendly solvents or MW activation.

A very interesting field of application is in the preparation of new organic materials and polymers. For instance, conjugated polymers, which are highly desirable for the development of organic semiconducting materials, ${ }^{[5]}$ are very often obtained using Stille polycondensations, involving coupling reactions of a symmetrical distannane with a dihalide compound. This transformation can be performed very easily as shown in Scheme 1, for the prepara-

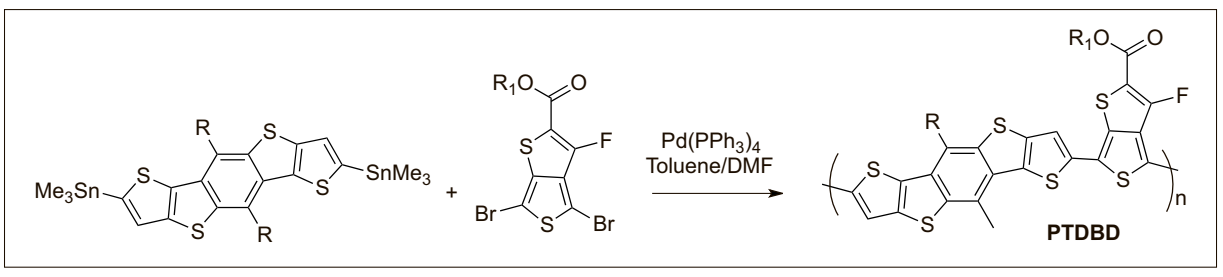

Scheme 1. Preparation of conjugated polymers via Stille polycondensation. 
Fig. 2. DSSC working mechanism.

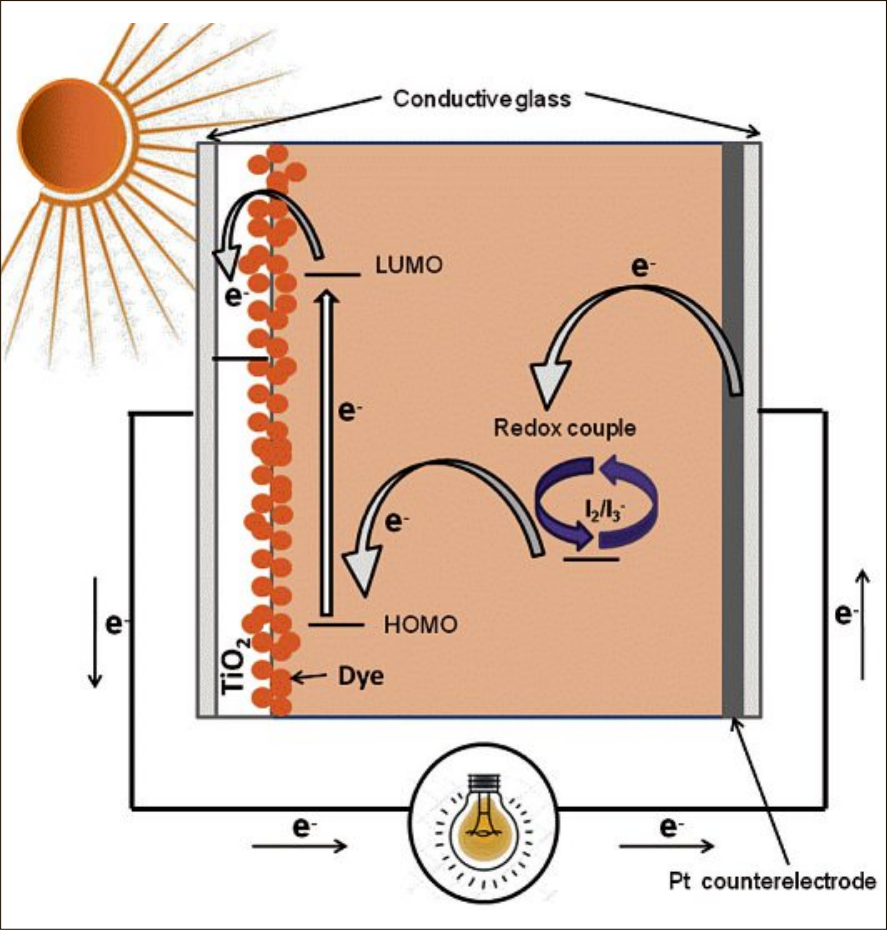

Fig. 3. Donor- $\pi$ acceptor conjugated $(D-\pi-A)$ structures.

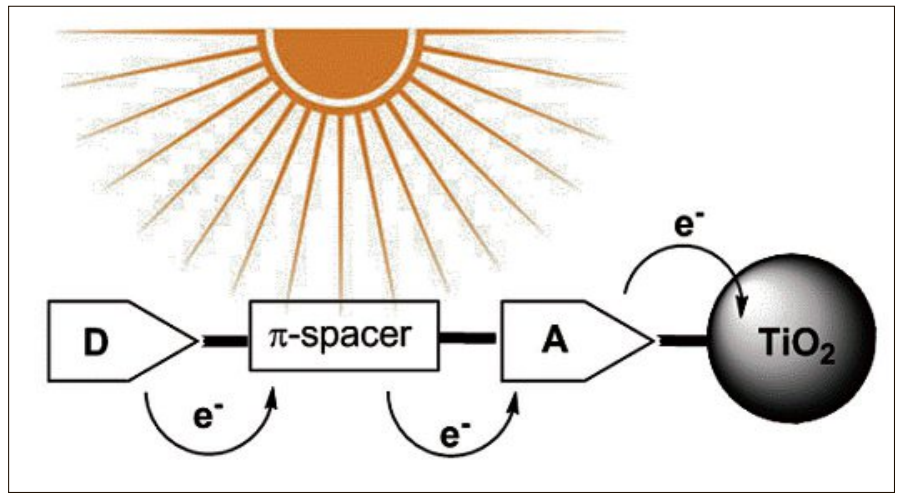

Meanwhile, the electron travels

tion of polymers of the PTDBD series, ${ }^{[6]}$ which are promising candidates for highperformance organic solar cells.

In the same area, another very recent and stimulating field in which the Stille reaction has found successful application is the design and synthesis of new photosensitizers for dye-sensitized solar cells (DSSCs). Among the new photovoltaic technologies, DSSCs are becoming a promising alternative for energy production from renewable sources; their working principle ${ }^{[7]}$ is inspired by natural photosynthesis, as light harvesting is carried out by a dye which is absorbed on a thin-layer of a mesoporous semiconductor (usually $\mathrm{TiO}_{2}$ ). The DSSC working mechanism is represented in Fig 2. Following photo-excitation, an electron is promoted from the HOMO of the dye to its LUMO, and from there is transferred to the conduction band of the semiconductor, $\mathrm{TiO}_{2}$. The original state of the dye is restored by electron donation from the electrolyte which is usually an organic solvent containing a redox system, such as iodide/triiodide redox couple. cathode (most often constituted by a thin layer of platinum), where the reduction of the oxidized redox mediator takes place, closing the circuit. Clearly the photosensitizer is a key component of such devices and for this reason many efforts have been devoted to the search of cheap, colorful, stable and highly efficient organic dyes for DSSCs, in order to increase the commercial attractiveness of this technology and establish it as a valid tool for solar energy exploitation.

Indeed, through the years a large number of organic dyes have been designed, synthesized and tested, ${ }^{[7 b, 8]}$ exhibiting in many cases high DSSC performances. One of the most promising classes of organic sensitizers is that of D- $\pi$-A conjugated structures, where an electron-rich donor group (D) is connected through a conjugated $\pi$-spacer to an electron-withdrawing acceptor (A), usually also responsible for the anchoring of the dye to the nanocrystalline semiconductor ${ }^{\text {[7b,8a] }}$ (Fig. 3).

The main interest of such structures is due to the fact that each part of the molecule can be strategically altered through chemical modification in order to tune the photophysical, electrochemical and photovoltaic properties of the dye. Indeed, such properties are strongly dependent on the electron-donating and the electronaccepting ability of the donor (D) and the acceptor (A) groups, respectively, as well as on the effective photo-induced intramolecular charge transfer which should be allowed by the $\pi$-conjugated spacer. In many cases, an arylamine moiety is used as the electron donor and 2-cyanoacrylic acid as the electron acceptor. This is due to the excellent stability, electron-donating ability and aggregation resistance of triphenylamine (TPA) and to the strong electron-withdrawing effect of cyanoacrylic moiety. In Fig. 4 some typical structures of organic D- $\pi$-A dyes are reported together with the power conversion efficiency measured for the corresponding DSSC devices $(\eta$, ratio between the elec-

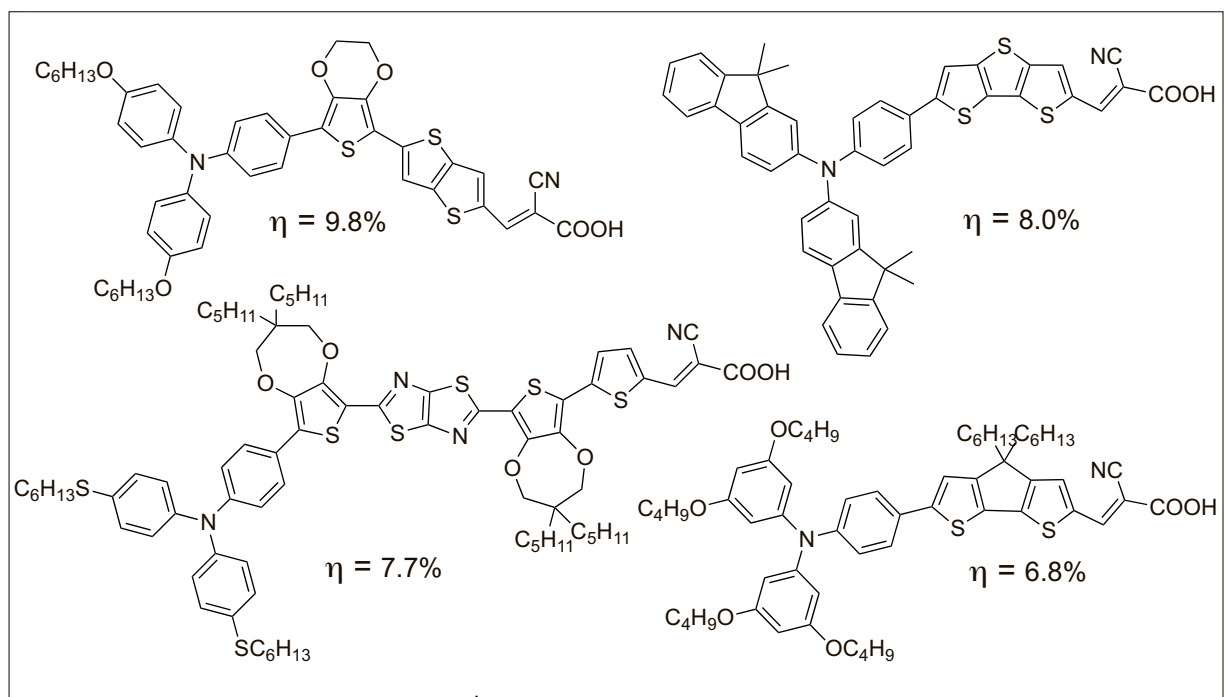

Fig. 4. Some examples of D- $\pi$-A organic dyes endowed with good efficiencies. 
tric power produced by the cell and the power of the incoming simulated solar radiation). ${ }^{[9]}$

To prepare this kind of highly conjugated structure, different approaches can be used. For example cross-coupling reactions, which are one of the most efficient and general methods for $\mathrm{C}-\mathrm{C}$ (sp) and $\mathrm{C}-\mathrm{C}\left(\mathrm{sp}^{2}\right)$ bond formation, have been widely exploited to build-up the $\pi$-scaffold, or introduce different donors. As we have already anticipated, the Stille cross-coupling reaction, due to the mild base-free reaction conditions and general scope, has been widely used for the assembly of DSSC organic sensitizers. In many instances, this procedure has been found superior to other cross coupling reactions, allowing the desired compounds to be obtained with higher yields and to introduce a wider molecular diversity, especially on large scale.

\section{Applications of Stille Couplings in the Synthesis of Organic Dyes for DSSC}

1,3,4-Oxadiazole-based compounds have been investigated as potential $\pi$-scaffolds for DSSCs dyes. For example, dye OD3, bearing the oxadiazole moiety, triphenylamine and $\pi$-bridged cyanoacrylic acid cores (Scheme 2), was designed and synthesized to act as donor- $\pi$-acceptor organic photosensitizer for dye-sensitized solar cells. ${ }^{[10]}$ The synthetic strategy involved a Stille cross-coupling between the corresponding oxadiazole bromide and 5-(tributylstannyl)thiophene-2-carbaldehyde, using standard conditions with $\mathrm{Pd}\left(\mathrm{PPh}_{3}\right)_{4}$ as the catalyst, to yield the key aldehyde intermediate, which was further condensed with cyanoacetic acid under Knoevenagel conditions. Photovoltaic measurements indicated the feasible use of compound OD3

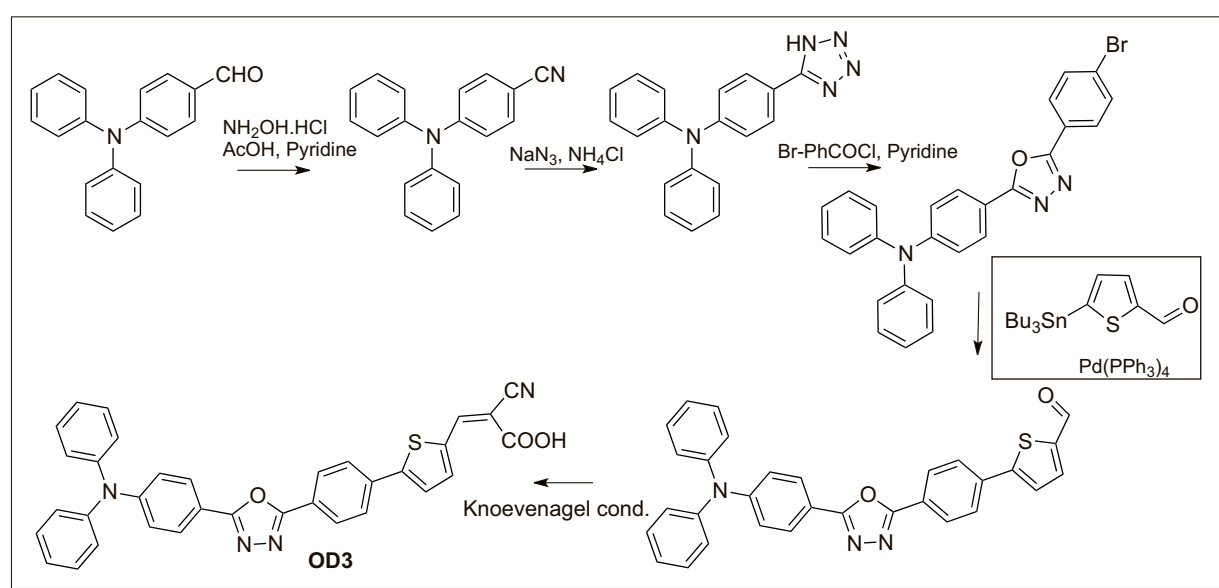

Scheme 2. Synthesis of a 1,3,4-oxadiazole dye via Stille cross coupling.

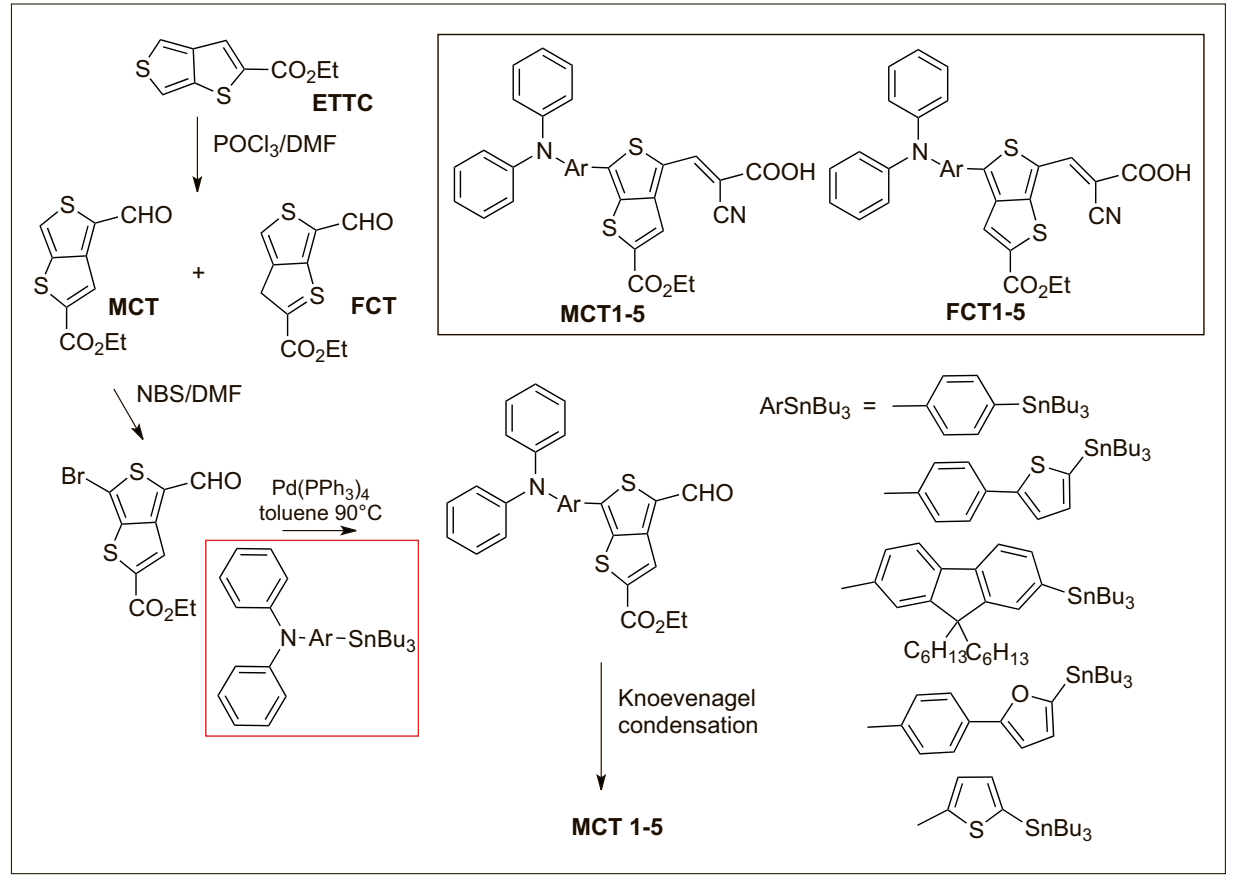

Scheme 3. Synthesis of ETTC-containing dyes. in dye-sensitized solar cells, although the final devices showed quite low efficiency.

Exploration of new sensitizers aiming to achieve a better correlation between structure and cell performance need the design of new classes of candidates, possibly having similar structures to allow a fair comparison. To this end, $[3,4-b]$ thiophene-2-carboxylate (ETTC) was inserted into the conjugated $\pi$-spacer of $D-\pi$-A structure. Formylation of ETTC can lead to two possible isomeric structures, MCT and FCT (Scheme 3), in which the sulfur atom of the thiophene-2-carboxylate is either closer to or more distant from the acceptor. ${ }^{[11]}$

Consequently, two series of dyes, MCT1-5 and FCT1-5, were prepared, which allowed a systematic study for comparison. Formylation of starting material (ETTC) via Vilsmeyer-Haack reaction led to two isomers, which were converted via electrophilic bromination with NBS into the corresponding bromo-formylthieno [3,4-b]thiophenes. These bromides are key intermediates to prepare both series of dyes through palladium-catalyzed Stille coupling. Reaction with the appropriate stannyl reagent provided the suitable formyl precursors which were transformed in the desired products MCT 1-5 (or FTT 1-5), via Knoevenagel condensation with cyanoacetic acid. Comparison of the photophysical properties of the two series of compounds showed that the MCT isomers have longer absorption wavelengths with weaker absorption intensity than the FTT isomers. Both MCT and FTT compounds exhibit bathochromic shift of the charge transfer absorption compared to their thiophene congeners, due to the more energetically favorable quinoid structure of ETTC compared to thiophene. When used as sensitizers for DSSCs, the best power conversion efficiency was found in the FTT series, with a maximum of $5.31 \%$.

DSSCs with promising photovoltaic performances have been obtained using dyes incorporating the coumarin ring system. ${ }^{[12]}$ In particular, some organic dyes containing [bis(9,9-dimethylfluoren-2-yl) amino]coumarin as electron donor and cyanoacrylic acid as electron acceptor bridged by thiophene units, named JK-34 and JK-35 (Scheme 4), have been prepared ${ }^{[13]}$ and used to build the corresponding DSSCs, which gave power conversion efficiencies up to $4.5 \%$.

Once again the key intermediates, the coumarin mono- and bis-thiophene protected aldehydes, were synthesized in good yields by coupling reaction of 7-amino3-bromo-4-methylcoumarin with stannylthiophene units in the presence of a catalytic amount of tetrakis(triphenylphosphine) palladium (0), according to the more classical Stille reaction conditions. 


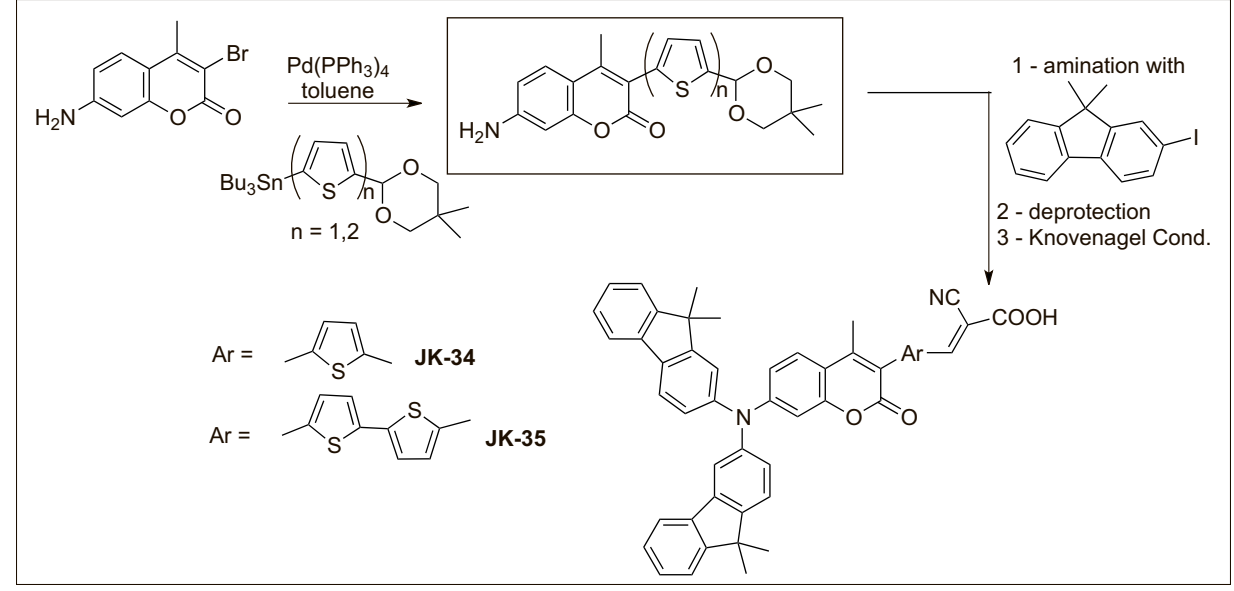

Scheme 4. Synthesis of coumarin-derived DSSC dyes.

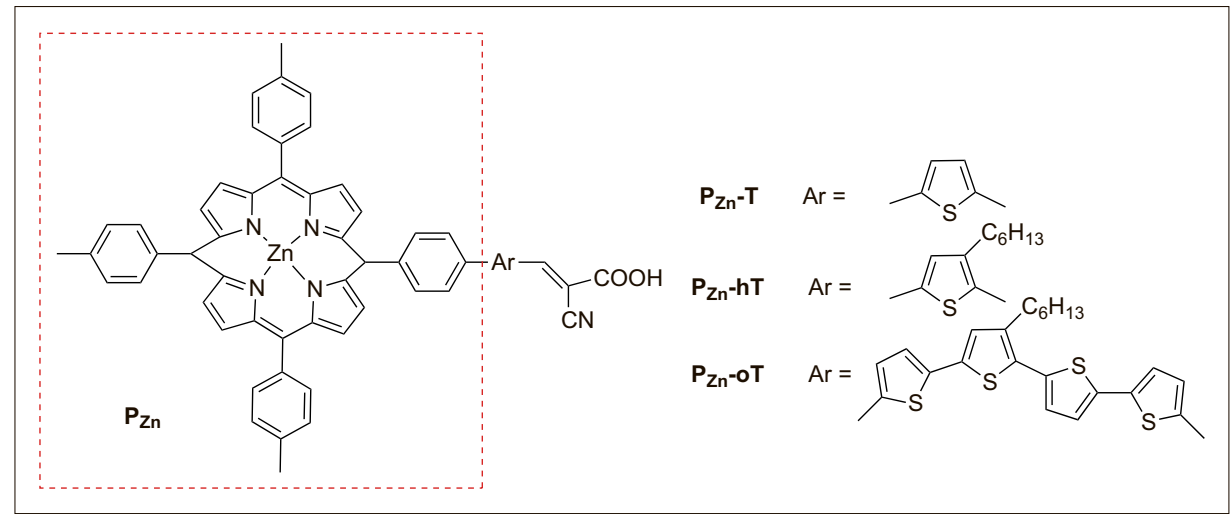

Fig. 5. Structures of porphyrins with extended $\pi$-conjugation.

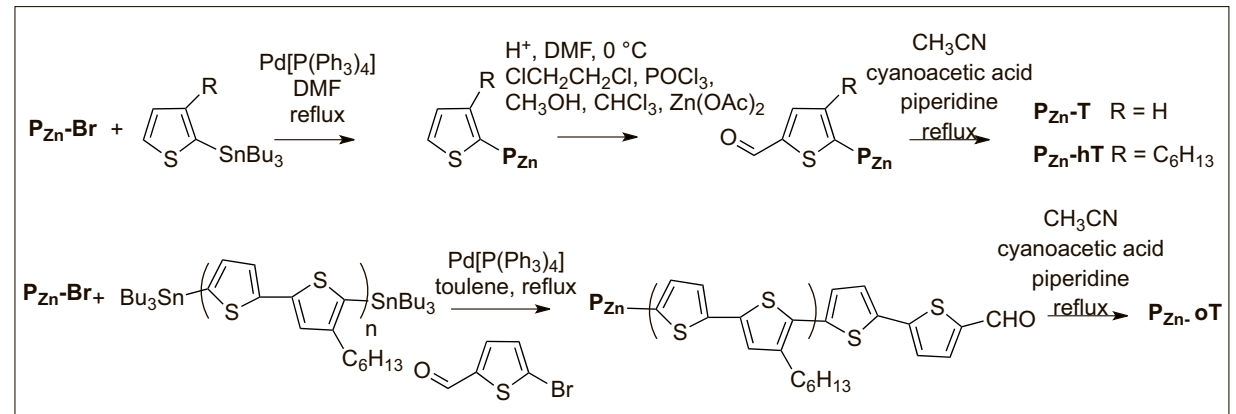

Scheme 5. Synthetic pathway to access thienyl porphyrins PZn-T, PZn-hT and PZn-oT.

Fig. 6. Molecular structures of dyes $\mathbf{S} 4$ and $\mathbf{S 5}$.
Among the organic dyes, porphyrins and their derivatives have also been studied as potential photosensitizers in DSSCs. In particular porphyrins with an extended $\pi$-conjugation proved able to give excellent photovoltaic performances. ${ }^{[14]}$ Once again, the Stille reaction emerged as a valid tool for porphyrin derivatization by elongation of their unsaturated scaffold.

As an example, dyes PZn-T, PZn-hT and PZn-oT have been prepared, in which the porphyrin unit and the cyanoacrylic acceptor/anchoring group were separated by thiophene rings, with the aim of broadening and strengthening their light-absorption characteristics (Fig 5). The effects on the photoelectrochemical and photovoltaic performances due to the increased number of thiophene rings (PZn-oT) or to adding a hexyl side chain (PZn-hT) of the dyes were systematically studied.

All dyes were indeed efficiently prepared using a Stille coupling: in the first two cases the bromide precursor PZn-Br was reacted in DMF under standard conditions using a suitable tributylstannylthiophene derivative. The corresponding aldehydes were then obtained by Vilsmeier formylation (Scheme 5). To prepare PZnoT the precursor PZn was reacted first with a tributylstannyloligothiophene, and subsequently with 5-bromothiophene-2-carbaldehyde. Finally, Knoevenagel reaction with cyanoacetic acid gave the desired porphyrin-containing dye (Scheme 5).

Among the three porphyrin dyes, cells built with PZn-hT exhibited the best power conversion efficiency with a maximum of $5.14 \%$. Spectroscopic data showed that indeed the $\pi$-conjugated thiophene-based central unit improved the light harvesting capability of the dyes, and its length, as well as the presence of an alkyl side-chain, could influence the electron transport efficiency, both of which are crucial for photovoltaic performance of DSSCs.

There are only few cases of organic bluecolored dyes reported for DSSCs, however very recently two organic blue-colored dyes have been prepared. ${ }^{[15]}$ Remarkably, such dyes featured high molar extinction coefficients and broad absorption spectra, which are essential to enhance the light harvesting efficiency.

Designed sensitizers S4 and S5 had a quite complex molecular structure which was based on a 2-ethylhexyl-substituted cyclopentadithiophene (CPDT) unit as the $\pi$-system, a indeno[1,2- $b]$ thiophene functionalized triphenylamine as strong electron donor, and a 2,3-diphenylpyrido[3,4$b$ ] pyrazine (PP) or 2,3-diphenylquinoxaline (QT) units, with a pronounced electron-withdrawing ability, as the auxiliary acceptors (Fig. 6). The alkyl chains were introduced to increase the solubility in organic solvents and minimize aggregation, 


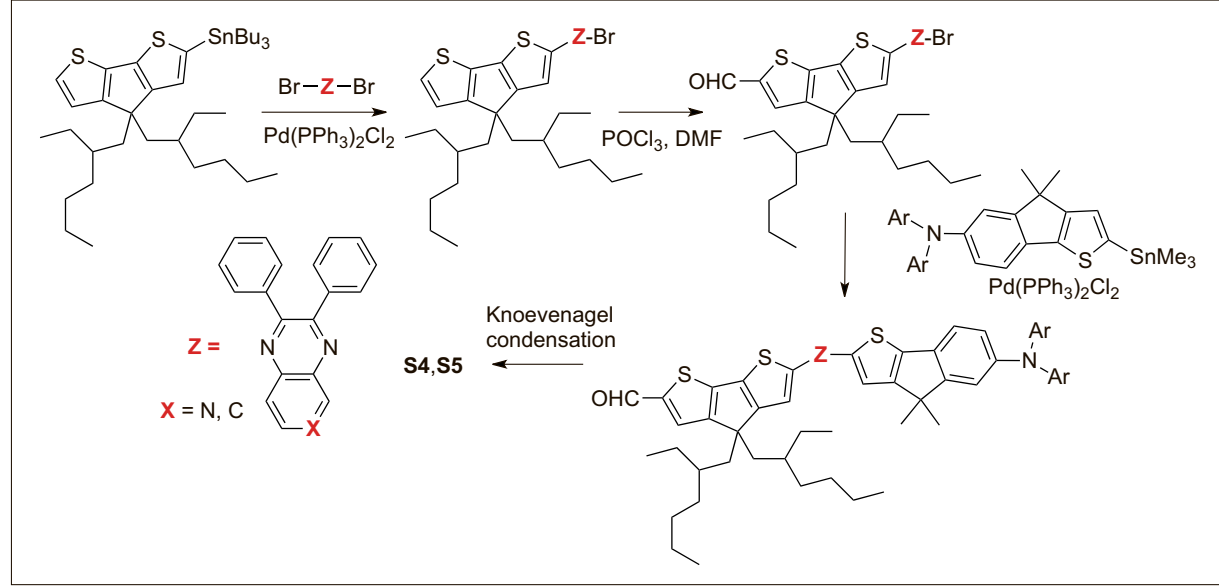

Scheme 6. Synthetic route of S4 and S5.

both in solution and upon adsorption on $\mathrm{TiO}_{2}$. To synthesize these dyes, two subsequent Stille couplings have been employed (Scheme 6)

First 5,8-dibromo-2,3-diphenylpyrido[3,4- $b]$ pyrazine or 5,8 -dibromo-2,3-diphenylquinoxaline have been reacted with $(4,4-b i s(2-e t h y l$ hexyl)-4Hcyclopenta[2,1- $b: 3,4-b]$ dithiophen-2-yl) tributylstannane to obtain the intermediate PP or QT bromides, which have been transformed into the corresponding aldehydes by a Vilsmeier-Haack reaction with $\mathrm{N}, \mathrm{N}$-dimethylformamide and phosphorus oxychloride. The second Stille coupling was used to insert the (CPDT) unit using the corresponding stannane. The target products S4 and S5 were then obtained following the usual Knoevenagel condensation with cyanoacetic acid in the presence of acetic acid and ammonium acetate. The two dyes showed interesting spectroscopic and photovoltaic performances, in particular dye S5 exhibited a remarkably high molar extinction coefficient of $6.30 \times 10^{4} \mathrm{M}^{-1}$ $\mathrm{cm}^{-1}$ at $600 \mathrm{~nm}$, and efficiency close to $8 \%$, which encourages further development of blue-colored dyes with high molar extinction coefficients for application in DSSCs.

3,4-Ethylenedioxythiophene (EDOT) fragment has been used widely in DSSC dyes as a conjugated bridge unit between the donor and acceptor residues due to its ability to facilitate co-planarity and favor light harvesting. ${ }^{[16]}$ This electron-rich moiety was introduced into the scaffold of a family of dyes based on the thiazolo[5,4-d] thiazole p-spacer, which was also endowed with bispentylpropylenedioxythiophene (ProDOT) moieties. The presence of bulky and electron-rich ProDOT groups besides the electron-poor thiazolothiazole unit imparted optimal physico-chemical properties to these dyes, including broad and intense visible light absorption. As a consequence, they were particularly suitable for application in thin layer-DSSCs. ${ }^{[9 b, 17]}$ To test the effect of adding the EDOT fragment in comparison with a simple thiophene unit, thiazolo[5,4- $d]$ thiazole dyes TTZ6 and TTZ3 (Fig. 7) were prepared and their spectroscopic characteristics and photovoltaic performances were compared.

To introduce the EDOT group, the appropriate stannane was prepared in a onepot procedure, starting from 2-formylethylenedioxythiophene, ${ }^{[18]}$ by protection with trimethylorthoformate followed by lithiation of the resulting dimethylacetal and treatment with tributyltin chloride; at the end of the reaction, work-up with an aqueous solution of $\mathrm{KHSO}_{4}$ led to deprotection of the formyl group, allowing isolation of the desired stannane in good yield (Scheme 7).

The EDOT-stannane was then coupled with the suitable iodide to afford compound TTZ6 in very good yield. Indeed, TTZ6 showed a red-shifted absorption maximum compared to TTZ3, although the efficiency of the corresponding cells were very similar. However, when photoanode sensitization was conducted in the presence of $1 \mathrm{mM}$ chenodeoxycholic acid (CDCA), which is generally used as a co- adsorbent to reduce dye aggregation on the semiconductor surface, no performance enhancement was observed in the case of TTZ6, perhaps due to the steric bulk of its terminal EDOT ring which can minimize aggregation even in the absence of CDCA.

Another critical issue in the design of new and efficient DSSC dyes is the choice of the anchoring group, which, besides being a good electron acceptor, should provide a good immobilization of the adsorbed dye molecules onto the semiconductor surface. In this way, the efficient injection of electrons from the dye into the conduction band of titanium oxide is promoted, together with a stable attachment of the dye onto the semiconductor, which is essential to ensure a long term stability to the devices. As stated above, the most frequently used anchors in DSSCs are cyanoacrylic acid groups which lead to a stable linkage between the dye and $\mathrm{TiO}_{2}$ by forming a strong bidentate bridge with titanium atoms. However, it has been shown that this functional group might undergo an undesirable retroaldolic degradation reaction under illumination and in the presence of traces of water, ${ }^{[19]}$ and this can negatively affect device lifetime. For this reason, several different anchoring groups have been proposed and tested by various researchers including phosphonate, sulfonate, catechol, siloxane and pyridine moieties.[20] In particular, carboxypyridine-derived anchoring groups showed very interesting features, which were highlighted when the photovoltaic performances and anchoring stability on $\mathrm{TiO}_{2}$ of different regioisomeric carboxypyridines were investigated in comparison with those of cyanoacrylic acid acceptor. ${ }^{[21]}$ To do that, the $\pi$-scaffold of the well-known dye D5[7b] (Fig. 8) was modified by introduction of three different carboxypyridines acceptors as anchor-
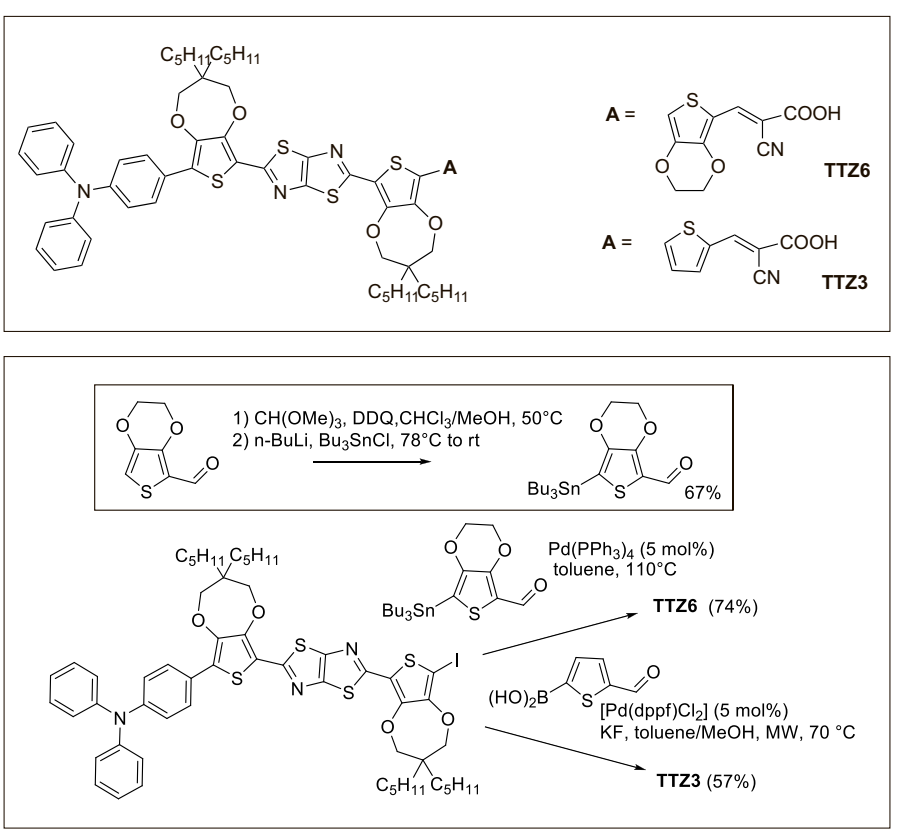

Fig. 7. Molecular structure of thiazolo[5,4-d]thiazole (TTZ) dyes.
Scheme 7. Synthetic route of thiazolo[5,4- $d$ ] thiazole dyes TTZ6 and TTZ3. 


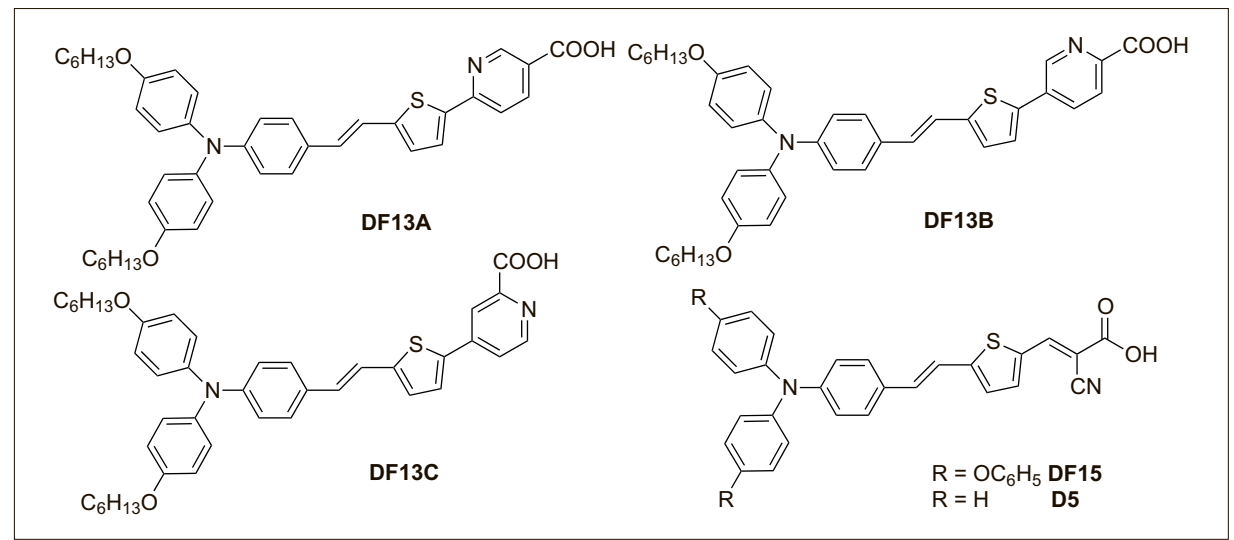

Fig. 8. Dyes with carboxypyridine-based acceptor moieties (DF13A-C) and cyanoacrylic derivatives D5, DF15.

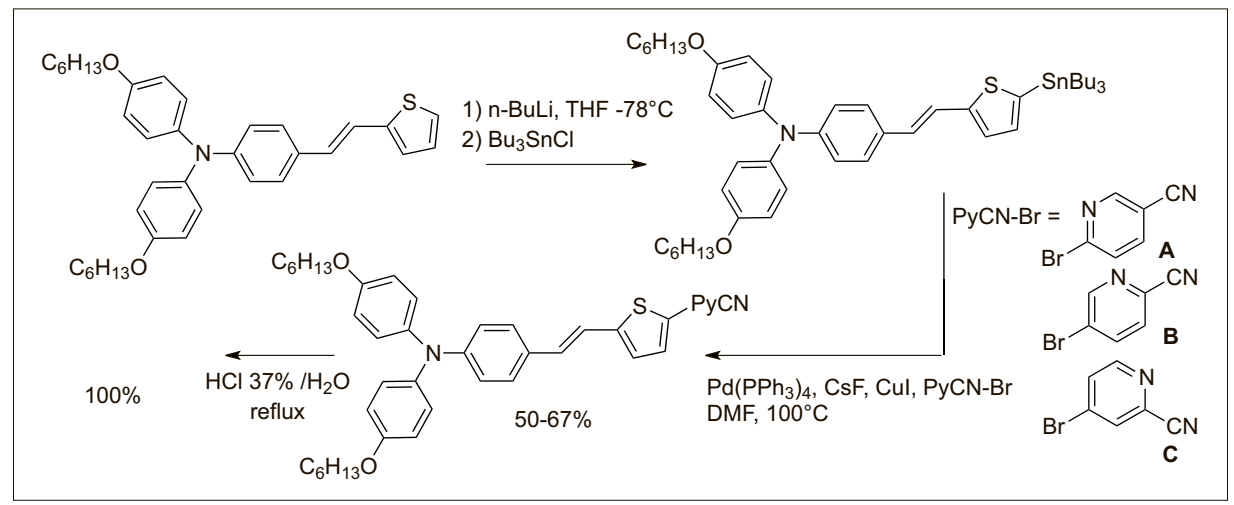

Scheme 8. Preparation of dyes DF13A,B,C.

ing groups, leading to preparation of dyes DF13A,B,C (featuring alkoxy chains on the triarylamine donor as well). Dye DF15, with the classic cyanoacrylic acid acceptor was also prepared for comparison.

Once again, the best way to prepare dyes DF13A,B,C was to transform a common advanced intermediate, thiophenylvinyl-triarylamine, ${ }^{[22]}$ into the corresponding stannane, and to couple it with regioisomeric bromocyanopyridines $\mathbf{A}, \mathbf{B}, \mathbf{C}$ (Scheme 8). The desired stannane was obtained simply by reaction with $n$-BuLi followed by quenching with $\mathrm{ClSnBu}_{3}$, and was reacted with bromopyridines using $\mathrm{Pd}\left(\mathrm{PPh}_{3}\right)_{4}$ as the catalyst, oxygen-free $\mathrm{DMF}$ as the solvent, $\mathrm{CsF}$ and $\mathrm{CuI}$ as additives. ${ }^{[23]}$ The corresponding cyanopyridines were obtained in good yields and transformed, after hydrolysis with concentrated aqueous $\mathrm{HCl}$, to the desired dyes DF13A,B,C in almost quantitative yield. It is worth mentioning that the same transformation was also attempted using a Suzuki-Miyaura cross-coupling between the corresponding pinacolboronic ester and the same bromopyridines, however it turned out to be less efficient than the Stille-Migita process described above, being affected by low yields and formation of side products. Dyes DF13A,B,C showed improved anchoring stability to $\mathrm{TiO}_{2}$ compared to DF15 and, in some cases, satisfactory photovoltaic performances, proving that introduction of carboxypyridine-derived anchoring groups can indeed represent a promising strategy to enhance DSSC lifetime and durability. ${ }^{[24]}$

\section{Conclusions}

The Stille reaction has been proved repeatedly to be a powerful tool for the preparation of new organic materials. In particular, it has found many successful applications in the design and synthesis of new photosensitizers for dye-sensitized solar cells (DSSCs). Such applications clearly show how the mild operating conditions and the high functional-group compatibility highlight this procedure as the best choice not only for the preparation of photosensitizers, but also to carry out their chemical variation in order to tune and optimize their photophysical, electrochemical and photovoltaic properties.

Received: July 28, 2017

[1] D. Milstein, J. K. Stille, J. Am. Chem. Soc. 1978, 100, 3636.

[2] a) A. F. Littke, L. Schwarz, G. C. Fu, J. Am. Chem. Soc. 2002, 124, 6343; b) S. P. Mee, V. Lee, J. E. Baldwin, Chem. Eur. J. 2005, 11, 3294; c) C. Cordovilla, C. Bartolomé, J. M. MartínezIlarduya, P. Espinet, ACS Catal. 2015, 5, 3040

[3] M. M. Heravi, E. Hashemi, F. Azimian, Tetrahedron 2014, 70, 7 .
[4] see for instance: H. Tang, K. Menzel, G. C. Fu, Angew. Chem. Int. Ed. 2003, 42, 5079.

[5] a) B. Carsten, F. He, H. J. Son, T. Xu, L. Yu, Chem. Rev. 2011, 111, 1493; b) C. Li, Z. Bo, 'New Chemistry for Organic Photovoltaic Materials', Royal Society of Chemistry, 2015; c) S. Xu, E. H. Kim, A. Wei, E. Negishi, Sci Technol. Adv. Mater. 2014, 15, 44201.

[6] H. J. Son, L. Y. Lu, W.Chen, T. Xu, T. Y. Zheng, B. Carsten, J. Strzalka, S. B. Darling, L. X. Chen, L. P. Yu, Adv. Mater. 2013, 25, 838.

[7] a) B. O'Reagen, M. Grätzel, Nature 1991, 353 , 737; b) A. Hagfeldt, G. Boschloo, L. Sun, L. Kloo, H. Pettersson, Chem. Rev. 2010, 11, 6595.

[8] a) C.-P. Lee, R. Y.-Y. Lin, L.-Y. Lin, C.-T. Li, T.C. Chu, S.-S. Sun, J. T. Lin, K.-C. Ho, RSC Adv. 2015, 5, 23810; b) Y. Wu, W. Zhu, Chem. Soc. Rev. 2013, 42, 2039; c) I. N. Obotowo, I. B. Obot, U. J. Ekpe, J. Mol. Struct. 2016, 1122, 80.

[9] a) N. Zhou, K. Prabakaran, B. Lee, S. Chang, B. Harutyunyan, P. Guo, M. Butler, A. Timalsina, M. Bedzyk, M. Ratner, S. Vegiraju, S. Yau, C.-G. Wu, R. Chang, A. Facchetti, M.-C. Chen, T. Marks, J. Am. Chem. Soc. 2015, 137, 4414; b) A. Dessì, M. Calamante, A. Mordini, M. Peruzzini, A. Sinicropi, R. Basosi, F. Fabrizi de Biani, M. Taddei, D. Colonna, A. Di Carlo, G. Reginato, L. Zani, Chem. Commun. 2014, 50, 13952; c) E. Gabrielsson, H. Ellis, S. Feldt, H. Tian, G. Boschloo, A. Hagfeldt, L. Sun, Adv. Energy Mater. 2013, 3, 1647.

[10] S. B. Mane, C.-F. Cheng, A. A. Sutanto, A. Datta, A. Dutta, C.-H. Hung, Tetrahedron 2015, 71, 7977.

[11] Y.-C. Chen, H.-H. Chou, M. C. Tsai, S.-Y. Chen, J. T. Lin, C.-F. Yao, K. Chen, Chem. Eur. J. 2012, 18,5430

[12] a) K. Hara, T. Sato, R. Katoh, A. Furube, Y. Ohga, A. Shinpo, S. Suga, K. Sayama, H. Sugihara, H. Arakawa, J. Phys. Chem. B 2003, 107, 597; b) K. Hara, M. Kurashige, Y. Dan-oh, C. Kasada, A. Shinpo, S. Suga, K. Sayama, H. Arakawa, New J. Chem. 2003, 27, 783.

[13] H. Choi, C. Baik, H. J. Kim, J.-J. Kim, K. Song, S. O. Kang, J. Ko, Bull. Korean Chem. Soc. 2007, 28, 1973.

[14] a) Q. Wang, W. M. Campbell, E. E. Bonfantani, K W. Jolley, D. L. Officer, P. J. Walsh, K. Gordon; R. H. Baker, M. K. Nazeeruddin, M. Graetzel, J. Phys. Chem. B 2005, 109, 15397; b) S. Hayashi, M. Tanaka, H. Hayashi, S. Eu, T. U. Meyama, Y. Matano, Y. Araki, H. Imahori, J. Phys. Chem. C 2008, 112, 15576; c) N. Koumura, Z. S. Wang, S. Mori, M. Miyashita, E. Suzuki, K. Hara, J. Am. Chem. Soc. 2006, 28, 14256; d) S. Eu, S. Hayashi, T. Umeyama, A. Oguro, M. Kawasaki, N. Kadota, Y. Matano, H. Imahori, J. Phys. Chem. C 2007, $111,3528$.

[15] Z. Shen, B. Xu, P. Liu, Y. Hu, Y. Yu, H. Ding, L. Kloo, J. Hua, L. Sunbe, H. Tian, J. Mater. Chem A 2017, 5, 1242.

[16] L. Li, C.-Y. Wang, R. Huang, M. R. Biscoe, Nat. Chem. 2013, 5, 607.

[17] A. Dessì, M. Calamante, A. Mordini, M Peruzzini, A. Sinicropi, R. Basosi, F. F. d. Biani, M. Taddei, D. Colonna, A. Di Carlo, G. Reginato, L. Zani, RSC Adv. 2015, 5, 32657.

[18] A. K. Mohanakrishnan, A. Hucke, M. A. Lyon, M. V. Lakshmikantham, M. P. Cava, Tetrahedron 1999, 55, 11745

[19] C. Chen, X. Yang, M. Cheng, F. Zhang, L. Sun, ChemSusChem 2013, 6, 1270.

[20] L. Zhang, J. M. Cole, ACS Appl. Mater. Interfaces 2015, 7, 3427.

[21] D. Franchi, M. Calamante, G. Reginato, L. Zani, M. Peruzzini, M. Taddei, F. F. d. Biani, R. Basosi, A. Sinicropi, D. Colonna, A. D. Carlo, A. Mordini, Tetrahedron 2014, 70, 6285.

[22] C. F. H. Allen, J. W. Gates, Org. Synth. 1945, 25, 9

[23] S. P. H. Mee, C.-R. Lee, J. E. Baldwin, Angew. Chem. Int. Ed. 2004, 43, 1132.

[24] C.-L. Mai, T. Moehl, C.-H. Hsieh, J.-D. Décoppet, S.M. Zakeeruddin, M. Grätzel, C.-Y. Yeh, ACS Appl. Mater. Interfaces 2015, 7, 14975 